\section{More Fun With Photoshop}

Tina Weatherby

University of Hawaii at Manoa

As humans, we are very sight oriented. As microscopists, we usually acquire data that is in the form of images. Since micrographs, or pictures, are what we deal with, we tend to become involved with the presentation of the data and its visual impact. When making a figure plate for publication, do you often find yourself moving images around, adjusting sizes, tonal gradations, and positions to achieve a more visually pleasing arrangement? Microscopy must be the most artistic of the sciences. We might like to have fun with our images, as well.

Images formed by electrons are collected on media that sees only if the electrons were there or not. Traditional electron micrographs are black and white images; photons are needed for the colors that we can detect. However, more and more often electron micrographs are colorized for presentation in the popular press in order to enhance details of what might otherwise be uninterpretable by lay persons. The use of color can help to point out details that might otherwise be difficult for the uninitiated to see. And, aw heck, colorizing scanning electron micrographs is just plain fun and lets the right side of the brain get some exercise!

Here's a quick primer for colorizing scanning electron micrographs, based on Photoshop 5.0 (but similar for versions 3.0 to 4.0 ).

Launch Photoshop. Your desktop should have at least a Menu Bar at the top of the window and a Toolbox consisting of a rectangle containing two columns of tool icons plus a few other controls. In addition, you may or may not see one or more Floating Palettes on the desktop. We'll get back to these. On the Menu Bar select File Open, then find and select the file name for your black and white image. Check on its size and resolution (Image - Image Size) and adjust if necessary.

Most electron micrographs need a little gamma correction, so go to Image - Adjust - Levels. You will see a grey levels histogram. Move the three arrows under the histogram until you get a pleasing image. For colorizing, a larger range of greys (gamma, the middle arrow) is best, but with the overall appearance a bit on the dark side for better color saturation.

Sharpen the image, if desired with Filter - Sharpen - Unsharp Mask.

Save your adjusted black and white image.

Now go to Image - Mode - RGB Color. This makes your file three times larger to accommodate the 3 color channels.

Now let's find the Floating Palettes if they are not showing on your desktop. Go up to the Menu Bar and click on Window to see a list of options. Four of the groups in the dropdown menu are palettes; click on Show Swatches to see what happens. A Floating Palette of small squares of color should appear. We are going to want to work with the palettes Options, Brushes, and Swatches. The Floating Palettes can be moved around and recombined by dragging their tabs: I usually combine Brushes and Options into one palette, and Color and Swatches into another, leaving the rest on Hide until I need them.

Double click on the Paintbrush icon in the Toolbox to get the Paintbrush Ooptions floating palette. Located in this palette are the choices for what are known as Blending Modes, but are not labeled as such. These choices appear in the dropdown menu just under the Paintbrush Options tab. Click on the down arrow and see the seven choices. Choose Color, the second to last one. This will allow you to paint over your black and white micrograph, applying color (hue) while retaining luminosity and saturation. In other words, all your detail will remain and only color will be applied.

Now choose a brush size. Find your Brushes palette (Windows - Show - Brushes). There are rows of both sharp and fuzzy spots, representing brushes of different sizes and hardnesses. The ones without numbers are shown actual size, and the numbers under the larger ones indicate pixel size. Click on the brush labeled 45 and then move the mouse over your image. If you get a cursor that is not a circle, go to the Menu Bar and choose File - Preferences - Display and Cursors. Select Brush Size for the Painting Cursors and you will change the cursor to a circle that denotes the size of the brush.

Finally, it's time to choose a color. There are two squares in the lower third of the rectangular Toolbox. They appear either in the colors last used or in black and white. The upper left square will be the foreground color, and the lower right will be the background color. Click on the foreground square and a window will appear with a large square containing various shades of some hue, a color slide bar, a smaller divided square, and a bunch of mysterious letters and numbers. Run the slider up and down the color bar to choose a hue, and use the large square to choose saturation and brightness. Click on Ok to put that color in the foreground square. Click on the background square and repeat the process for another color.

Alternatively, you may choose colors from the floating palettes Color and Swatches. Foreground and background colors in the Toolbox may be toggled by clicking on the little curved arrow to their upper right.

Now drag your mouse over your image while holding the button down for Macs, or the left button for PCs. You should have succeeded in painting color over your image without obscuring any of the detail. If it didn't work, check the Options floating palette to make sure that you are in the Color blending mode with the Opacity at $100 \%$ and Fade at 0 .

Now you are ready to paint. It's just like coloring in a coloring book! Are you going outside of the lines too much? Increase the image display size one or more times with View - Zoom In. Note what the keyboard shortcuts are in that drop down menu so that you can use them instead of the mouse to zoom in and out if you wish. Try a smaller, hard-edged brush for outlining, and then switch to a larger fuzzy brush to fill in.

Still going outside of the lines? Perhaps you would like to use the selection tools in the Toolbox so that only the areas you select are operated on. There are a number of these; the Rectangular Marquee, the Elliptical Marquee, Single Row and Single Column tools, the Lasso, the Polygonal Lasso, the Magnetic Lasso (version 5.0 only), the Magic Wand, and the Pen. Once chosen, each of these tools works somewhat differently, and I will leave it to your explorations to figure them out. Once an area is selected, you can drag your paintbrush all over the image but color will only "stick" inside the selected areas.

Here's an example of how this might work. Say you have an SEM image of an insect on a fairly plain background. In this case it's easiest to select the background. Double click on the Magic Wand in the Toolbox to see its Options in a floating palette. Make sure Anti-aliased is checked, then enter a number in the Tolerance box. Tolerance is the range of brightness values of pixels that will be included in the selection. In other words, if you choose a tolerance of 12 and click on your image, all pixels with a 
value within a range of 12 (on a scale of 0 to 255) that are contiguous with the value of the image pixel you clicked on will be selected. If you choose 56 you will get a lot more pixels selected than if you choose 4 . The point is to select the background without selecting parts of your bug. With experience you will be able to guess a good Tolerance value. For now, choose 12 .

Click on the largest background area of your image. A moving dotted line outlines the selected area. Only a tiny area selected? Increase your Tolerance. Too much of your bug included? Decrease Tolerance. To add more areas of background to the selection, hold down the Shift key and click on another part of the background. To subtract an area from the selection, hold down the Alt key (Option key on Macs). Continue to add (shift-click) or subtract (alt-click or option-click) until the entire background is selected. Now if you paint an area of your image, the paint will only be applied to the background.

Want to paint the bug instead? On the Menu Bar go to Select - Inverse, and now you will be able to paint the bug and not the background. The Lasso is another great tool, allowing you to select an area with the mouse freehand. The Lasso and other selection tools also make use of the Shift key to add and Alt (Option) key to subtract.

If you do something you don't like, immediately go up to the top bar and select Edit - Undo. This will only undo the very last thing you did before releasing the mouse button. Do you hate everything you did since the last Save (you do remember to save frequently, don't you?), choose File - Revert. If you only wish to undo the last few operations, make sure you have Photoshop 5.0 and use the History palette to get rid of unwanted portions.

Explore the tools and operations and you will find ways to alter the hue, saturation, brightness, contrast, curves and levels There are a multitude of ways to color and change your image. Dig out your old Microscopy Today (October '97), find "Fun with Photoshop", and create new worlds. Just remember to go back to your regular life as a microscopist tomorrow morning!

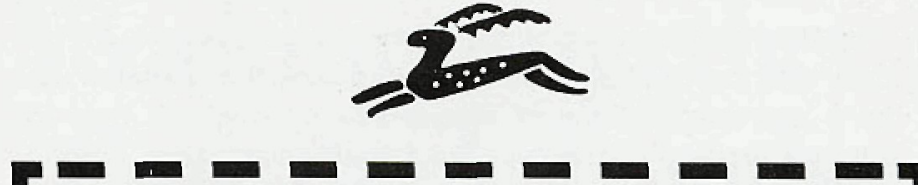

Best Quality, Best Price Microscope Bulbs

Osram Mercury HBO 100, only $\$ 107.99$

Osram Mercury HBO 103 , only $\$ 116.99$

Osram Xenon XBO75 Ozone Free, only $\$ 132.99$

2 bulb minimum - add $\$ 5.00$ per bulb for single orders

Shipping/handling/insurance from stock only $\$ 4.95$

Telephone or On-Site Technical Support Upon Request: $\$ 42.50$

Best Quality, Best Price Objective Repair

Inside/Outside Oil Removal per Objective only $\$ 59.99$

If not repairable, shop fee only $\$ 24,99$

Return shipping/handling/insurance $\$ 14.95$

\section{Sciscope Instrument Company Leica Kodak Sony Osram Dealer \\ Mail orders: Box 2237, lowa City, lowa 52244 USA USA telephone orders: $1-800-55-\mathrm{MICRO}$ \\ Overseas telephone orders: $+319-338-1107$}

Fax orders: 1-319-338-3954 Internet orders: www.sciscope.com

* Include your Visa, Master Card or American Express information

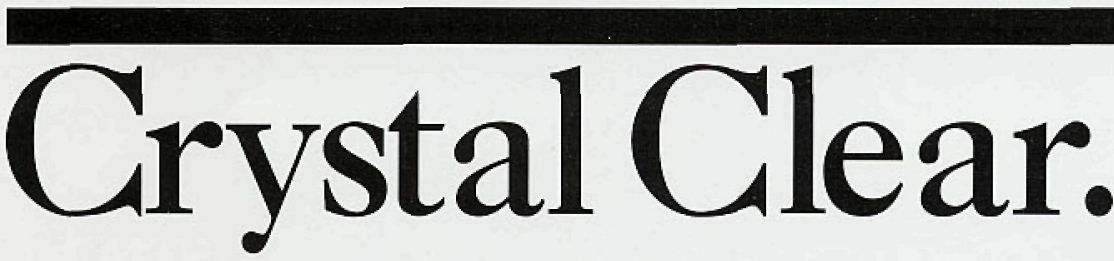

\section{The Meiji EM Series of Modular Stereo Mierosbopes.}

If you are looking for precision, durability, quality and value in a stereo microscope, we invite you to take a closer look at Meiji's EM Series of Stereo Microscopes.

The modular design (A wide variety of bodies, single magnification or zoom - rotatable $360^{\circ}$, auxiliary lenses, eyepieces, stands, holders, etc.) gives you the freedom to create the ideal instrument for your specific needs or application, and Meiji stands behind every instrument with its limited Lifetime Warranty.

For more information on these economically priced stereo microscopes, please call, FAX or write us today.

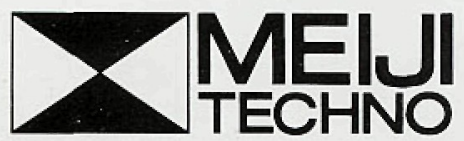

\section{MEIJI TECHNO AMERICA}

2186 Bering Drive, San Jose, CA 95131, Toll Free Telephone: 800.832 .0060 FAX: 408.428.0472, Tel: 408.428.9654 